\title{
Abordagem adaptativa aplicada ao planejamento agregado da produção sob incertezas
}

\author{
Oscar Salviano Silva Filho ${ }^{\mathrm{a}, *}$, Wagner Cezarino ${ }^{\mathrm{b}}$ \\ a,*oscar.salviano@cti.gov.br, CTl, Brasil \\ bwagner.cezarino@cti.gov.br, CT1, Brasil
}

\begin{abstract}
Resumo
Um problema de planejamento agregado da produção, com incertezas sobre a flutuação da demanda, é formulado através de um modelo de otimização estocástica, com critério quadrático e restrições lineares. Dificuldades para encontrar uma solução ótima global para o problema levam à proposição de uma abordagem adaptativa de fácil implementação computacional que é baseada na formulação de um problema determinístico equivalente e que tem sua solução periodicamente revisada por meio de um procedimento clássico da literatura. Um exemplo, em que o sistema de balanço de estoque está sujeito à forte e fraca variabilidade da demanda real, é empregado para analisar o comportamento da abordagem proposta. Por fim, os resultados obtidos são comparados com outra abordagem subótima, cuja principal característica é não permitir revisões periódicas.
\end{abstract}

Palavras-chave

Controle de estoques. Planejamento. Otimização. Processos estocásticos. Simulação.

\section{Introdução}

0 artigo considera um problema de planejamento agregado da produção sujeito a flutuações aleatórias da demanda, que é formulado a partir de um modelo de otimização estocástica com restrições nas variáveis de decisão. 0 objetivo é produzir um plano de produção - constituído por níveis ótimos esperados de estoque, produção, subcontratação, mão de obra regular e hora extra - que possa ser revisado periodicamente pela administração da empresa.

Uma solução ótima global para este tipo de problema, via de regra, é muito difícil de ser obtida pelas técnicas clássicas de programação matemática e teoria de controle ótimo, incluindo também o algoritmo de programação dinâmica estocástica; (CHENG et al., 2004; ORTEGA; LIN, 2004; BERTESEKAS, 2000). As causas desta dificuldade são basicamente as seguintes: (1) incertezas relacionadas à flutuação de demanda; (2) alta dimensionalidade do problema; e (3) restrições que impõem limites físicos a serem satisfeitos. Como resultado, soluções aproximadas, desenvolvidas a partir de procedimentos subótimos - que abrem mão do ótimo global -, são muito frequentes na literatura. A justificativa para utilização de abordagens subótimas é que elas são mais fáceis de programar e processar computacionalmente do que aquelas que buscam soluções globais, como é o caso do algoritmo de programação dinâmica.

É importante destacar que a maioria das abordagens subótimas tem duas características comuns, a saber: a primeira é que, usualmente, utilizam o princípio da equivalência à certeza (BERTESEKAS, 2000) para reduzir a complexidade do problema estocástico. Este princípio estabelece que todas as variáveis aleatórias do problema estocástico devem ter seus valores fixados em seus respectivos primeiros momentos estatísticos. Como consequência, o problema estocástico original é transformado diretamente em um equivalente determinístico, conhecido como Problema da Média. A segunda diz respeito à utilização de algum esquema de realimentação para atualizar periodicamente a política ótima de decisão. Com isto, é possível afirmar que a precisão dessas abordagens depende da quantidade de informação absorvida ao longo dos períodos de tempo.

Com base no exposto acima, propõe-se neste trabalho uma abordagem composta de duas partes principais, a saber: na primeira, o modelo estocástico é aproximado por um equivalente determinístico que preserva suas propriedades originais. Isto 
significa que a convexidade do critério, a linearidade das restrições e os dois primeiros momentos estatísticos das variáveis aleatórias do problema são mantidos na formulação. Como consequência, o problema equivalente pode ser visto como uma extensão ao Problema da Média e será denominado aqui de Problema Estendido da Média (PEM). A segunda parte da abordagem consiste em adotar algum procedimento da literatura, que permita gerar soluções revisadas para o PEM. Dentre os inúmeros procedimentos subótimos disponíveis (KLEINDORFER, 1978), optou-se pelo procedimento OLU (Open Loop - Updating) em razão de sua simplicidade de implementação computacional, que segue um esquema equivalente ao da técnica conhecida como horizonte deslizante (PEREIRA; SOUSA, 1997). Como resultado, obtém-se uma abordagem adaptativa (i.e. que permite ajustes no tempo) de simples implementação e que gera políticas de melhor desempenho, a custos menores.

É importante aqui destacar que se, durante a solução do PEM, nenhuma medida do estado real do sistema de balanço de estoque for permitida, a solução ótima gerada é dita ser estática, ou seja, que não pode ser corrigida no tempo. Este tipo de solução é resultado da aplicação de um procedimento subótimo conhecido na literatura como Open Loop - No-updating (OLN) o qual considera o estado inicial do sistema como única informação disponível para solução do PEM, qualquer informação futura disponível é totalmente ignorada (KLEINDORFER, 1978).

Para validar a abordagem aqui proposta e, simultaneamente, comprovar sua característica adaptativa, propõe-se, através de um exemplo, que sua solução ótima seja comparada àquela produzida pelo procedimento OLN, aplicado diretamente ao PEM. Para isto dois cenários produtivos serão criados, nos quais o sistema de balanço de estoque estará sujeito, respectivamente, à fraca e forte variabilidade na flutuação da demanda real. Em ambos os cenários, espera-se mostrar que o plano gerado com a abordagem proposta apresenta um desempenho melhor do que o plano gerado por uma abordagem que não utiliza um mecanismo de revisão no tempo, como é o caso do procedimento OLN.

0 artigo está distribuído como se segue: na seção 2, o problema de otimização estocástica com restrições probabilísticas é formulado e sua notação é introduzida. Em seguida, a seção 3 apresenta uma breve revisão da literatura, contemplando assuntos relacionados com a solução de problemas de planejamento agregado da produção. Já a seção 4 discute o problema determinístico equivalente, resultante do problema estocástico formulado na seção 2. Baseado nessa formulação, discute-se a aplicação do procedimento OLU, formalizando-se assim a abordagem proposta. Por fim, a seção 5 apresenta um exemplo de uso dessa abordagem.

\section{Planejamento agregado da produção: modelo estocástico}

Um plano ótimo de produção - desenvolvido para satisfazer as flutuações de demanda de um dado produto, no horizonte de planejamento de T períodos de tempo - é descrito por uma sequência ótima de variáveis de decisão $\left\{x_{i}^{*}, u_{i}^{*}, v_{i}^{*}, w_{i}^{*}, z_{i}^{*} ; i=1, \ldots, T\right\}$, resultante da solução do problema de otimização estocástic a:

$$
\underset{u}{\operatorname{Min} J} J c_{x} E\left\{x_{T}^{2}\right\}+\sum_{t=1}^{T-1}\left\{c_{x} E\left\{x_{t-1}^{2}\right\}+c_{u} u_{t}^{2}+c_{v} v_{t}^{2}+c_{w} w_{t}^{2}+c_{z} z_{t}^{2}\right\}
$$

S.a.

$$
\begin{aligned}
& x_{t-1}-x_{t}+u_{t}+v_{t}=d_{t}, \quad x_{0}=E\left\{x_{0}\right\} \\
& t_{p} \cdot u_{t} \leq p_{d} \cdot\left(w_{t}+z_{t}\right) \\
& -t_{p} \cdot u_{t} \leq\left(p_{s}-1\right) \cdot w_{t} \\
& z_{t} \leq p_{h} \cdot w_{t} \\
& \operatorname{Prob} .\left(x_{t} \geq S_{s}\right) \geq \alpha ; \operatorname{com} \alpha \in[0,1) \\
& 0 \leq u_{t} \leq C_{m} ; 0 \leq v_{t} \leq B_{s} ; 0 \leq w_{t} \leq Q_{r} ; 0 \leq z_{t} \leq H_{r}
\end{aligned}
$$

cujas variáveis e parâmetros são denotados como se segue:

\subsection{Variáveis de decisão}

- $x_{\mathrm{t}}=$ quantidade de produto a ser deixada em estoque no período $t$;

- $u_{\mathrm{t}}=$ quantidade de produto a ser produzida durante o período $t$;

- $v_{\mathrm{t}}=$ quantidade de produto subcontratado para complementar estoque do período $t$;

- $w_{\mathrm{t}}=$ quantidade de mão de obra regular escalada para atuar no período $t$, e

- $Z_{\mathrm{t}}=$ quantidade de mão de obra regular adicional em atividade de hora extra no período $t$.

\subsection{Demanda temporal e parâmetros do modelo}

- $d_{\mathrm{t}}=$ nível de demanda real (i.e., quantidade de produto efetivamente consumida) no período $t$. 
É uma variável aleatória com distribuição de probabilidade conhecida;

- $p_{\mathrm{d}}=$ percentagem permitida da capacidade de produção, para eventuais paradas, devido à manutenção programada, set-ups de máquina, além de fatos inesperados como quebra de maquinário;

- $t_{\mathrm{p}}=$ percentual de tempo de processamento para produzir uma unidade do produto;

- $p_{\mathrm{h}}=$ fração da quantidade de mão de obra regular usada de forma equivalente ao percentual de hora extra permitido pela empresa;

- $p_{\mathrm{s}}=$ percentual mínimo de mão de obra regular efetivamente utilizado;

- $C_{\mathrm{m}}=$ capacidade máxima de produção;

- $S_{\mathrm{s}}=$ estoque de segurança previamente fixado para o produto;

- $B_{\mathrm{s}}=$ quantidade máxima do produto a ser subcontratado de terceiros;

- $Q_{\mathrm{r}}=$ quantidade máxima de mão de obra regular disponível; e

- $H_{\mathrm{r}}=$ quantidade máxima de mão de obra regular adicional em atividade de hora extra.

\subsection{Parâmetros do critério}

$c_{x}, c_{u}, c_{v}, c_{w}$ e $c_{z}$ são constantes que denotam os preços relacionados, respectivamente, com os custos de armazenagem, produção, subcontratação, mão de obra e hora extra.

Algumas características importantes a destacar do problema estocástico (1a-g) são:

1) Existem, essencialmente, três variáveis de decisão independentes, descritas por $\left\{u_{t}, v_{t}, w_{t}\right\}$, que devem ser determinadas conjuntamente a partir de seus respectivos espaços restritivos, definidos em (1g). Já as variáveis $x_{t}$ e $z_{t}$ são ditas variáveis de decisão dependentes, pois devem ser especificadas automaticamente a partir dos valores ótimos obtidos para $\left\{u_{t}, v_{t}\right\}$ e $w_{t}$, respectivamente. É interessante observar que este conjunto de variáveis de decisão já é suficiente para inviabilizar o uso do algoritmo de programação dinâmica estocástica, pois o uso de três ou mais variáveis de decisão já conduz à maldição da dimensionalidade; vide Powell (2007). Como consequência, obter uma solução ótima global para o problema $(1 \mathrm{a}-\mathrm{g})$ a partir de recursos computacionais ordinários, ou seja, aqueles que não envolvem computação paralela ou algo equivalente, é totalmente inviável (GRAMA et al., 2003).

2) A demanda real $d_{t}$ é uma variável aleatória independente e estacionária que representa os níveis agregados de consumo do produto ao longo de cada período de tempo t. Segundo Graves (1999), o comportamento estacionário da demanda permite que sua distribuição de probabilidade possa ser aproximada por um modelo de distribuição normal. Assim sendo, a demanda $d_{t}$ pode ser completamente caracterizada por sua média histórica e variância finita, dadas, respectivamente, por $\mu_{d}$ e $\sigma_{d}^{2} \geq 0$.

3) 0 sistema linear de balanço de estoque, equação (1b), deve ser interpretado como um processo estocástico. Isto decorre do fato do nível de demanda ser uma variável aleatória que, a cada novo período de tempo, contamina a evolução dinâmica deste sistema. Devido à linearidade deste sistema, a função distribuição de probabilidade da variável de estoque é similar à distribuição de probabilidade da demanda, com média e variância perfeitamente identificadas no tempo.

4) 0 critério (1a) é dado por uma combinação de funções quadráticas. Este tipo de custo, popularizado a partir do modelo de regra de decisão linear desenvolvido por Holt et al. (1960), tem sido muito explorado na literatura, não só por sua simplicidade mas também por permitir uma solução que penaliza tanto os níveis elevados (excesso) quanto os reduzidos (falta) das variáveis de decisão (HAX; CANDEA, 1984).

5) As restrições (1c) e (1d) definem relações de dependência entre a quantidade de mão de obra (em atividade regular ou em hora extra) e o nível de produção desejado, para operar o sistema (1b) a cada período de tempo $t$. Já a restrição (1e) define um limite máximo para uso de mão de obra em atividades de hora extra que é uma função direta da quantidade disponível de mão de obra regular.

6) A restrição (1f) representa o limite mínimo de estoque que deve ser mantido do produto ao longo do tempo. Ela é tomada em probabilidade em virtude do nível de estoque $x_{t}$, para cada período de tempo $t$, ser uma variável aleatória. 0 índice probabilístico $\alpha$ denota o nível de serviço esperado. Assim, por exemplo, assumindo $\alpha \cong 100 \%$, a empresa está tentando garantir o máximo de satisfação do cliente quanto à entrega nos prazos previamente combinados. Para este fim, ela aumenta os níveis de estoque de segurança principalmente próximo aos períodos finais do horizonte de planejamento. Por outro lado, assumindo $\alpha \cong 0$, a empresa trabalha com a hipótese de manter os níveis de estoque de segurança nos níveis previamente especificados pela administração (i.e. S) e, assim, confia no poder de negociação com os clientes, caso ocorram aumentos inesperados nos níveis de demanda em períodos futuros que possam ocasionar atrasos de entrega do produto.

7) Por fim, a restrição (1g) representa, respectivamente, os limites mínimos e máximos de capacidade de 
produção, de produção subcontratada; de mão de obra regular e adicional (i.e. em hora extra).

Uma solução ótima global para o problema (1) é uma tarefa muito complexa em virtude da natureza dinâmica estocástica do sistema de balanço de estoque (1b) e de suas restrições associadas. Além disso, a dimensionalidade do problema, que envolve cinco variáveis de decisão, inviabiliza o emprego do algoritmo de programação dinâmica estocástica. Como resultado, o uso de abordagens aproximativas (i.e., subótimas), que reduzem a complexidade do problema original, tem sido o caminho mais adotado na literatura.

\section{Breve revisão da literatura e contribuição}

Apenas a título de contextualização do tema tratado neste trabalho, escolheram-se, a partir da literatura, alguns artigos que têm relação direta com o problema de planejamento da produção com enfoque estocástico. A ideia é apresentar de uma forma sucinta as afinidades dos assuntos tratados nestes trabalhos com a abordagem aqui proposta. Segue-se então, de forma cronológica, uma exposição resumida de alguns desses artigos:

- Kleindorfer et al. (1975) discutem e demonstram que é possível desenvolver planos de produção aplicando abordagens da Teoria de Controle Ótimo Estocástico em problemas de planejamento agregado da produção. Eles discutem o uso de heurísticas subótimas como um caminho que simplifica o problema estocástico sequencial de modo a viabilizá-lo computacionalmente;

- Pekelman e Rausser (1978) discutem a importância do uso da informação no processo de tomada de decisão e apresentam um conjunto de heurísticas que tomam vantagem da informação disponível do sistema para gerar políticas ótimas de produção para problemas de planejamento de produção estocásticos sequenciais;

- Bitran e Yanasse (1984) consideram um problema de planejamento da produção estocástico com uma condição de nível de serviço. Estes autores propõem formulações determinísticas equivalentes para o modelo e determinam limites de erros entre as soluções estáticas (não sequenciais) e as soluções exatas geradas;

- Feiring e Sastri (1990) analisam um plano de produção com estratégias de horizonte deslizante e níveis de satisfação do cliente fixados pelos gerentes. As restrições probabilísticas do modelo são transformadas em restrições determinísticas, assumindo a hipótese da demanda ser normalmente distribuída;
- Shen (1994) analisou três métodos de controle ótimo estocástico. Esses métodos foram aplicados na solução de um problema determinístico equivalente formulado com base no modelo linear-quadrático de Holt et al. (1960). Dois dos métodos estudados incorporam medidas dos níveis de estoque observadas diretamente no sistema de produção (via algum esquema de realimentação de informação) para desenvolver a política subótima de produção;

- Hackman et al. (2002) consideram um problema de planejamento da produção estocástico sequencial em que o interesse é determinar quando disponibilizar produtos acabados para atender à demanda prevista. Na solução, os autores consideram níveis de qualidade de serviço definidos a partir das restrições de chance da variável de estoque e adotam um esquema de horizonte deslizante para promover ajustes periódicos na política de produção;

- Yildirim et al. (2005) analisam um problema de planejamento da produção através de um modelo sequencial estocástico com restrições de chance, que são transformadas em indicadores de nível de serviço ao cliente. Uma abordagem alternativa de solução do problema, baseada no procedimento de horizonte deslizante, é proposta pelos autores;

- Rantala e Koivisto (2006) apresentam um algoritmo para geração de um plano agregado, a partir de um problema de planejamento da produção estocástico sequencial, que leva em conta requisitos de qualidade de serviço; e

- Levi et al. (2005) discutem, na mesma linha dos autores anteriores, modelos e técnicas aproximativas para lidar com problemas de planejamento da produção, procurando melhorar a solução gerada a partir de revisões periódicas.

Analisando os trabalhos apresentados acima, é possível destacar algumas características consideradas pelos autores na análise e solução de problemas com natureza estocástica, a saber: a) simplificação do problema estocástico, permitindo que abordagens clássicas da literatura possam ser aplicadas; b) utilização de mecanismos de realimentação para ajustar a variável de decisão em relação ao estado do sistema; c) adoção de esquemas do tipo horizonte deslizante para atualização da solução do problema com respeito à disponibilidade de informação no período de estudo; e d) fixação de critérios para melhoria do nível de satisfação do cliente.

0 uso simultâneo de algumas características apresentadas nos modelos expostos acima pode ser visto como uma possível contribuição deste trabalho. De fato, a abordagem aqui proposta inclui: a) um modelo matemático equivalente que 
preserva as principais características estruturais e propriedades do modelo original, tais como, por exemplo: os dois primeiros momentos estatísticos das variáveis aleatórias, a linearidade das restrições e convexidade do critério; b) a fixação de níveis de serviço associados à política de entrega, permitindo assim a aplicação das melhores práticas de competitividade; c) um procedimento subótimo que permite resolver interativamente o modelo equivalente, produzindo um plano ótimo revisado. Além de um plano continuamente atualizado para empresa, através do uso desta abordagem é possível construir cenários ótimos de produção, os quais permitem à gerência estudar diferentes alternativas de como lidar com as flutuações de demanda.

\section{Sobre a abordagem proposta}

Nesta seção, uma abordagem quase ótima de solução para o problema (1) será apresentada. Basicamente, ela combina um modelo determinístico equivalente com um esquema interativo de solução baseado no procedimento Open Loop - Updating.

\subsection{Formulação do problema estendido da média (PEM)}

Em geral, um modelo determinístico equivalente é derivado a partir da aplicação do princípio da equivalência à certeza, o qual estabelece que uma aproximação razoável para um problema não determinístico é tomá-lo na média de suas variáveis aleatórias (BERTESEKAS, 2000). Este modelo equivalente é frequentemente denominado de Problema da Média. Neste estudo, no entanto, um modelo equivalente mais geral, que inclui, além da média das variáveis aleatórias, também suas respectivas variâncias, será proposto. Este modelo é denominado aqui de Problema Estendido da Média (PEM).

0 processo de conversão do problema original (1) ao equivalente PEM é muito simples, uma vez que é inteiramente baseado nas características estatísticas do sistema (1b), que é um processo assumido como normalmente distribuído. Com isto, a variável aleatória de estoque $x_{t}$ tem uma função distribuição de probabilidade, definida aqui por $\Phi_{x, t}$, que é completamente especificada a partir da evolução de seus dois primeiros momentos estatísticos, como se segue:

$$
\left\{\begin{array}{l}
\hat{x}_{t}=\hat{x}_{t-1}+u_{t}+v_{t}-\hat{d}_{t} ; \hat{x}_{t}=E\left\{x_{0}\right\} \\
\operatorname{Var}\left(x_{t}\right)=\sigma_{d}^{2} \cdot \sum_{j=1}^{t} j ; \operatorname{Var}\left(x_{0}\right)=0
\end{array}\right\}
$$

onde $\hat{x}=E\left\{x_{t}\right\}$ denota o nível médio de estoque e $\operatorname{Var}\left(\mathrm{x}_{t}\right)=E\left\{x_{t}^{2}\right\}-E\left\{x_{t}\right\}^{2}$ sua variância. Note-se que $\hat{d}_{t}$ é a demanda média projetada (i.e. demanda estimada) para cada período de tempo $t$, que tem como base a média histórica $\mu_{d}$ e o desvio padrão $\sigma_{d}$ da demanda real $d_{f}$.

Assim, pode-se observar que, para cada período de tempo $t$, toda a informação necessária sobre 0 estado da variável aleatória $x_{t}$ estará definida pelo par $(\hat{x},(\operatorname{Var} x))$. Com base em (2) é possível transformar o problema (1) no PEM, obtendo-se assim:

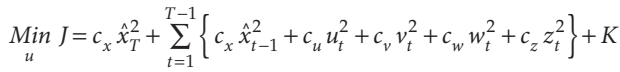

s.a.

$\hat{x}_{t-1}-\hat{x}_{t}+u_{t}+v_{t}=\hat{d}_{t}, \hat{x}_{0}=E\left\{x_{0}\right\}$

$t_{p} \cdot u_{t} \leq p_{d} \cdot\left(w_{t}+z_{t}\right)$

$-t_{p} \cdot u_{t} \leq\left(p_{s}-1\right) \cdot w_{t}$

$z_{t} \leq p_{h} \cdot w_{t}$

$\hat{x}_{t} \geq \underline{x}_{\alpha, t}=S_{s}+\Phi_{t}^{-1}(\alpha) \cdot \sqrt{t} \cdot \sigma_{d}$

$0 \leq u_{t} \leq C_{m} ; 0 \leq v_{t} \leq B_{s} ; 0 \leq w_{t} \leq Q_{r} ; 0 \leq z_{t} \leq H_{r}$

onde $K=\sigma_{d}^{2} \cdot \sum_{t=1}^{T} t$ denota o custo associado ao risco devido às flutuações de demanda. Este custo não influencia na solução ótima do problema (3), sua importância é só dar mais precisão ao custo total gerado. 0 limite mínimo de estoque $\underline{X}_{\alpha, t}$ é uma função do tempo $t$ e do nível de serviço desejado $\alpha$, sendo possível verificar que, fixado o valor de $\alpha$, tem-se que $\underline{X}_{\alpha, t+1} \geq \underline{X}_{\alpha, t}$, para $t=1,2, \ldots$, T. Isto significa que, para cada período de tempo $t$, a quantidade $\Phi_{t}^{-1}(\alpha) \cdot \sqrt{t} \cdot \sigma_{d}$ é adicionada ao nível do estoque de segurança $S_{s}$. Com isto, o objetivo é prevenir contra possíveis excessos de demanda ou atrasos inesperados de fornecedores, especialmente próximo aos períodos finais do horizonte de planejamento. Como discutido na seção 2 , o administrador pode usar o índice probabilístico $\alpha$ para estabelecer diferentes níveis de serviço ao cliente e, assim, analisar e escolher cenários apropriados para rodar seu plano de produção (SILVA FILHO; CEZARINO, 2007).

Por fim, é interessante observar que o PEM é uma generalização do Problema da Média. De fato, sempre que $\sigma_{\mathrm{d}} \approx 0$ implica que $\hat{d} \approx \mu_{\mathrm{d}}$ e, assim, como consequência, o PEM torna-se igual ao Problema da Média. 


\subsection{Esquema interativo de solução do PEM}

A abordagem proposta segue o procedimento OLU, o qual trabalha em estágios, denotados aqui pelo inteiro $k$. Cada estágio é coincidente com os períodos de tempo $t \in[1, T-1]$ do horizonte de planejamento $T$. Deste modo, a cada início de um novo período de tempo $t$, inicia-se também um novo estágio. Como resultado, no estágio $k$, o problema (3) será resolvido para faixa de tempo $j \in[1, T-t]$, com o estoque inicial $\hat{x}_{\mathrm{j}}=\mathrm{x}_{\mathrm{t}-1}$ medido no início do período $t$.

Em resumo, para cada estágio $k$, tão logo seja conhecido o estoque inicial $x_{t-1}$, a sequência ótima de produção, dada pelas variáveis de decisão independentes $\left\{u_{j}^{*}, v_{j}^{*}, w_{j}^{*}, j=1, \ldots, T-t\right\}$, é calculada resolvendo o problema (3), ligeiramente reformulado, como se segue:

$$
\begin{aligned}
& \underset{u}{\operatorname{MinJ}_{t}=}{ }_{c} \hat{x}_{T-t+1}^{2}+\sum_{j=1}^{T-t}\left\{c_{x} \hat{x}_{j-1}^{2}+c_{u} u_{j}^{2}+c_{v} v_{j}^{2}+c_{w} w_{j}^{2}+c_{z} z_{j}^{2}\right\}+K_{T-t} \\
& \text { s.a. } \\
& \quad \hat{x}_{j-1}-\hat{x}_{j}+u_{j}+v_{j}=\hat{d}_{j}, \hat{x}_{j}=x_{t-1} \text { (valor medido) } \\
& t_{p} \cdot u_{j} \leq p_{d} \cdot\left(w_{j}+z_{j}\right) \\
&-t_{p} \cdot u_{j} \leq\left(p_{s}-1\right) \cdot w_{j} \\
& z_{j} \leq p_{h} \cdot w_{j} \\
& \hat{x}_{j} \geq \underline{x}_{\alpha, j}=S_{s}+\Phi_{j}^{-1}(\alpha) \cdot \sigma_{d} \cdot \sqrt{j} \\
& 0 \leq u_{j} \leq C_{m} ; 0 \leq v_{j} \leq B_{s} ; 0 \leq w_{j} \leq Q_{r} ; 0 \leq z_{j} \leq H_{r}
\end{aligned}
$$

onde o custo $K_{T-t}=\sigma_{d}^{2} \cdot \sum_{j=1}^{T-t} j$ é pré-fixado para de cada estágio k e, como consequência, não altera as trajetórias ótimas de decisão. Da sequência ótima gerada $\left\{u_{j}^{*}, v_{j}^{*}, w_{j}^{*}, j=1, \ldots, T-t\right\}$ para o estágio $\mathrm{k}$, somente a componente inicial, dada em $j=1$, ou seja, $\left\{u_{k}=u_{1}{ }^{*} ; v_{k}=v_{1}^{*} ; w_{k}=w_{1}{ }^{*}\right\}$, será efetivamente utilizada. A sequência restante, definida para $j=2,3$, ..., $T-t$, é totalmente descartada. A razão disso é que ela é uma sequência estática no tempo (i.e., malhaaberta), não recebendo, portanto, nenhum tipo de atualização ao longo dos períodos restantes.

Terminado o estágio $\mathrm{k}$, o esquema prossegue para o estágio $k+1$. Isso significa que, no início do período $t+1$, tão logo seja feita uma nova medição no nível de estoque (i.e., $x_{j}=x_{t+1}$ ) diretamente do sistema de balanço de estoque (1b), que é totalmente

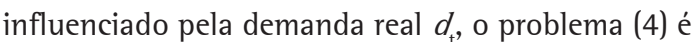
novamente resolvido, considerando-se agora a faixa de planejamento $j \in[1, T-t-1]$.

Como resultado, o esquema é repetido num total de $T-1$ vezes, quando, por fim, uma solução ótima revisada, descrita por variáveis de decisão independentes $\left.\left\{u_{k}^{*}, \quad v_{k}^{*}, \quad w_{k}^{*}\right\}\right|_{k=1, \ldots,-1} \mathrm{e}$ dependentes $\left\{x_{T}^{*} ;\left.\left(x_{k}^{*}, Z_{k}^{*}\right)\right|_{k=1 \ldots T-1}\right.$, é completamente gerada. Note-se que a variável de estoque ótima $x_{k}^{*}$ é resultado da aplicação direta das políticas de produção $\left\{u_{k}^{*}, v_{k}^{*}\right\}$ no sistema de balanço de estoque da empresa, e também que o custo ótimo final de aplicação desta política é dado por $\mathrm{J}_{o}^{*}{ }_{l u}=\mathrm{c}_{X} \hat{X}_{T}^{* 2}+$ $\sum_{k=1}^{T-1}\left\{c_{x} \hat{x} k+c_{u} u_{k}^{* 2}+c_{v} v_{k}^{* 2}+c_{w} w_{k}^{* 2}+c_{z} z_{k}^{* 2}\right\}$.

Neste ponto, é interessante mencionar a existência de outros procedimentos subótimos na literatura que também podem ser aplicados ao modelo do problema descrito em (4), vide, por exemplo, Bertesekas (1976). Estes procedimentos seguem a mesma natureza dinâmica do OLU, no entanto, podem ser mais ou menos eficientes na condição de captar informações do sistema real. Em outras palavras, quanto mais informações puderem ser incorporadas ao modelo, mais adaptativo será o procedimento e melhor será sua solução ótima. Assim, para compararmos o desempenho de diferentes procedimentos, deve-se aplicar o princípio da adaptatividade (BERTESEKAS, 1976), que estabelece que o custo ótimo gerado por cada procedimento está restrito a dois extremos, a saber: um é o custo ótimo global do tipo malha fechada $\left(J_{G \text { LOBAL }}^{*}\right.$, que é gerado pelo algoritmo de programação dinâmica estocástica; e o outro é o custo ótimo do tipo malha aberta $\left(J_{O}^{*} L N\right)$, que é gerado pelo procedimento OLN (Open-Loop No-updating) através de algum método aplicável da programação matemática. Deste modo, pelo princípio da adaptatividade, o custo ótimo gerado pelo OLU pertencerá à faixa $J_{G L O B A L}^{*}<J_{O}^{*} \angle U<J_{O}^{*}{ }_{L N}$. Prosseguindo, se for adotado outro Procedimento Subótimo $(P S)$ da literatura, que adapte ao modelo mais informações do sistema real que o OLU, pelo princípio da adaptatividade, chega-se a algo como $J_{G \text { LOBAL }}^{*}<J_{P S}^{*} \leq J_{O}^{*}{ }_{L U}<J_{O L N}^{*}$. Com respeito a este assunto, é importante destacar aqui duas coisas: primeira, que não está nos objetivos deste trabalho comparar a metodologia OLU com outras existentes, pois há trabalhos na literatura que fazem este tipo de análise com mais propriedade. Um bom exemplo, para interessados, é consultar o capítulo 3 em Bertesekas (1976); e segunda, que a natureza adaptativa do procedimento OLU pode ser conferida pelo leitor na seção 5, na qual o custo ótimo gerado pela aplicação do procedimento OLU é comparado ao custo gerado pelo procedimento OLN. Nos dois cenários investigados observa-se que $J_{O L U}^{*}<J_{O}^{*}{ }^{*}$. 


\subsection{Representação abordagem proposta}

esquemática

da

A Figura 1 ilustra como funciona a abordagem proposta que combina o procedimento OLU com a formulação PEM e permite a geração de cenários de planos de produção. É possível observar nesta Figura, que o problema (4) é resolvido apenas quando a informação sobre o estado real do sistema torna-se disponível num dado período $\mathrm{t}$ (i.e., $\hat{x}_{j}=\mathrm{x}_{t--}$ ). Da sequência ótima gerada, a taxa de produção $u_{k}=u_{i}^{*}$ e a quantidade subcontratada $v_{k}=v_{1}^{*}$ são as variáveis consideradas como entradas para o sistema de balanço de estoque. Este esquema permite que o plano gerado seja revisado a cada novo período de tempo $t$. Assim é possível garantir a realização de ajustes nas quantidades produzidas, subcontratada e de mão de obra sempre que os níveis de flutuação de demanda vierem a afetar o sistema de balanço de estoque ao longo de cada período $k=t$ do horizonte de planejamento $T$.

Note-se que, nesta abordagem, para cada estágio $k=t$, a demanda real $d_{t}$ é dada por informações extraídas do mercado no período $t$. 0 sistema de balanço de estoque é então colocado em operação com as entradas ótimas $u_{k}, v_{k}$ obtidas resolvendo-se o PEM. Na solução deste problema, adota-se uma demanda projetada $\hat{d}_{j}$ que é conhecida a priori para a faixa $j \in[t, \ldots, T-1]$. Esta demanda projetada é resultado de uma estimativa a longo prazo, baseada na expectativa média do comportamento da série temporal da demanda real $d_{f}$. Do sistema de balanço de estoque, é então conhecido o estado atual $x_{k}$ que será utilizado como condição inicial para gerar as variáveis de decisão independentes $\left\{u_{k+1}^{*}, v_{k+1}^{*}, w_{k+1}^{*}\right\}$ relacionadas ao estágio $k+1$, e assim por diante.

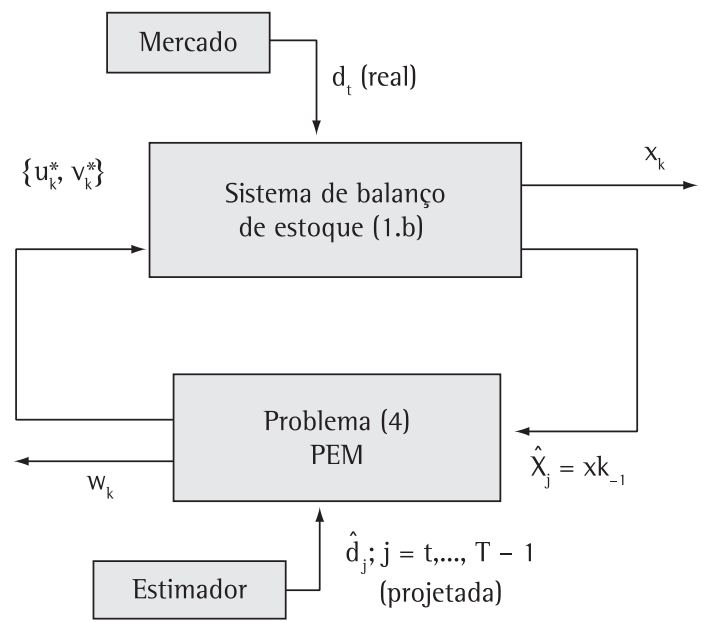

Figura1. Esquema da abordagem proposta para o estágio k=t.

\subsection{Geração de cenários via abordagem proposta}

0 esquema da Figura 1 mostra que a abordagem proposta pode ser utilizada na geração de cenários para a definição de planos agregados de produção, adequados aos interesses da empresa. Neste sentido, é importante observar o comportamento da demanda real $d_{t}$ e da demanda projetada $\hat{d}_{j}$ Por exemplo, no caso da demanda real, um gerador aleatório deve ser definido. Este gerador deve seguir uma distribuição de probabilidade pré-definida do tipo Exponencial, de Poisson ou mesmo Gaussiano, como é o caso neste estudo. Já a demanda projetada deve ser definida antecipadamente pelo projetista, usando algum mecanismo de estimação que utiliza dados históricos da série temporal da demanda real.

\section{Exemplo}

Considera-se uma empresa que produz uma linha de produtos do tipo commodity, empregado por várias empresas de transformação, e, portanto, sujeito a flutuações de demanda. É assumido que a evolução da demanda real agregada destes produtos, ao longo do tempo, segue um padrão cíclico-estacionário - vide definição e aplicação em Gardnera et al. (2006). Isto significa que ela evolui em ciclos periódicos em torno da média, tendo variância constante finita. Estes dois momentos estatísticos são predefinidos a partir de dados históricos disponíveis. A variabilidade cíclica de flutuação da demanda tem explicação no comportamento competitivo do mercado.

A empresa deseja desenvolver um plano agregado de produção de horizonte semestral que possa ser mensalmente revisado. Para esse fim, será empregada a abordagem proposta na seção 4. Com o objetivo de validar o uso da abordagem, dois cenários distintos de produção serão desenvolvidos, a saber: o primeiro cenário considera uma situação em que ocorrem variações de pequena intensidade na magnitude dos níveis de flutuação da demanda real; enquanto que, no segundo cenário essas variações são bem mais intensas. A ideia, então, é verificar de que forma estas flutuações têm impacto no plano agregado gerado. Além disto, as trajetórias ótimas das variáveis de decisão e os custos associados obtidos pela abordagem proposta serão comparados com aqueles gerados na aplicação do procedimento OLN ao PEM. 


\subsection{Dados do problema}

Os parâmetros básicos do problema são apresentados a seguir. É importante, antes, destacar que alguns destes parâmetros são aproximações de um estudo de caso da literatura relacionado com um problema de planejamento hierárquico da produção proposto em Özdamar et al. (1998). Para este exemplo tem-se que: a) o percentual de perda de capacidade de produção é fixado em $p d=10 \%$; b) o tempo de processamento ( $t p)$ é estimado em torno de 5\% de um turno de trabalho; c) a fração da mão de obra regular usada adicionalmente em atividades de hora extra é de $p_{h}=20 \%$; d) o percentual de mão de obra regular utilizado no processo de produção não deve ser inferior a $o s=90 \%$; e) o estoque de segurança está fixado em $S_{s}=50$ unidades e os limites máximos de produção e subcontratação foram fixados, respectivamente, em $C_{m}=110$ e $B_{s}=50$ unidades; e f) os limites máximos de mão de obra regular e mão de obra adicional são dados, respectivamente, por $Q_{r}=45$ homem/mês e $H r=9$ homem/mês. Outras informações de interesse são: g) o horizonte de planejamento é de seis meses (i.e. $T=6$ meses) com períodos mensais começando a partir do mês de janeiro e terminando no mês de junho. 0 estoque inicial medido no início de janeiro é de 90 unidades (i.e., $x_{0}=90$ ); h) a equipe de operação efetivamente contratada (i.e. mão de obra regular) trabalha em turnos diários de oito horas; i) é assumido que não existe atraso de produção no final dos seis meses; j) a demanda real $d_{t}$ segue um padrão tipo ciclo-estacionário, com média histórica igual a $\mu_{d}=120$ unidades e variância finita $\sigma_{d}^{2} \geq 0$, a ser especificada posteriormente durante a definição dos cenários; e l) os custos com manutenção de estoques, capacidade de produção, subcontratação de terceiros, mão de obra regular e adicional são assumidos por funções quadráticas e dados por:

$$
J=40 \cdot \check{x}_{6}^{2}+\sum_{t=1}^{T=6}\left\{40 \cdot \check{x}_{t-1}^{2}+5 \cdot u_{t}^{2}+15 \cdot v_{t}^{2}+10 \cdot w_{t}^{2}+27 \cdot z_{t}^{2}\right\}
$$

Por fim, assume-se que o nível de serviço adotado pela empresa é de 95\%. lsto significa que o índice probabilístico da restrição de estoque (3f) é $\alpha=0,95$. Com isto, a empresa pretende garantir "algo próximo" à máxima satisfação dos clientes quanto aos prazos de entrega.

\subsection{Análise de cenários}

Com base nos dados acima, planos agregados de produção podem ser gerados por meio da abordagem proposta, que utiliza o procedimento OLU, e do procedimento OLN aplicado diretamente ao PEM. Estas abordagens foram implementadas usando as funções matemáticas da plataforma MATLAB versão 5.0, em que, por exemplo, a solução do problema (4) utilizou a rotina de programação quadrática (i.e., "qp.m”).

A seguir dois cenários de produção são simulados usando o esquema da Figura 1. A flutuação da demanda real $\mathrm{d}_{t}$ é aleatória e é gerada pela expressão: $d_{t}=\mu_{d}+\sigma_{d} \delta_{t} \cdot \cos (\pi \cdot t)$, onde a variável $\delta_{t}$ é um ruído branco normal (PAPOULIS, 1991). Este tipo de demanda segue um comportamento conhecido como cíclico estacionário, que é irregular devido à perturbação branca $\delta_{t}$ inserida. Por outro lado, a demanda projetada, que é uma estimativa da demanda real, segue um padrão cíclico regular baseado na média histórica da demanda real e seu desvio padrão, definido aqui como $\hat{d}_{t}=\mu_{d}+\sigma_{d}$ $\delta_{t} \cdot \cos (\pi . t)$. No primeiro cenário assume-se que a demanda real tenha um desvio padrão finito de $\sigma_{d}=4$ unidades; enquanto que no segundo cenário o desvio é cinco vezes maior, ou seja, com $\sigma_{d}=20$ unidades. Note-se que, no primeiro, cenário o coeficiente de variabilidade é de apenas 3,33\%; enquanto, no segundo, este coeficiente é de $16,7 \%$. Isto significa que a previsibilidade do comportamento da demanda real é bem maior no primeiro cenário.

Por fim, é importante destacar que, para efeito de aplicação do procedimento OLN, o comportamento da demanda real foi assumido ser previamente conhecido. Com isto, na solução do PEM via OLN, utilizou-se a demanda real ao invés da projetada. Já com relação à abordagem proposta, o esquema da Figura 1 foi fielmente seguido, ou seja, a demanda real é conhecida somente a cada novo período de tempo e é usada para atualizar o sistema de estoque e consequentemente a política ótima gerada a partir da solução do PEM, que utiliza a demanda projetada no processo de solução.

\subsubsection{Cenário 1: Flutuação de demanda com desvio padrão $\sigma_{d}=4$}

Neste cenário, a variabilidade da flutuação de demanda em torno da média histórica é bastante suave, o que pode ser medido pelo baixo percentual do coeficiente de variação em torno de 3\%. Uma implicação disto é que a demanda projetada fica muito próxima da demanda real. A Figura 2a ilustra o comportamento da demanda real (linha tracejada) e da demanda projetada (linha cheia), oscilando suavemente em torno da média histórica $\mu_{d}=120$ (linha pontilhada). 

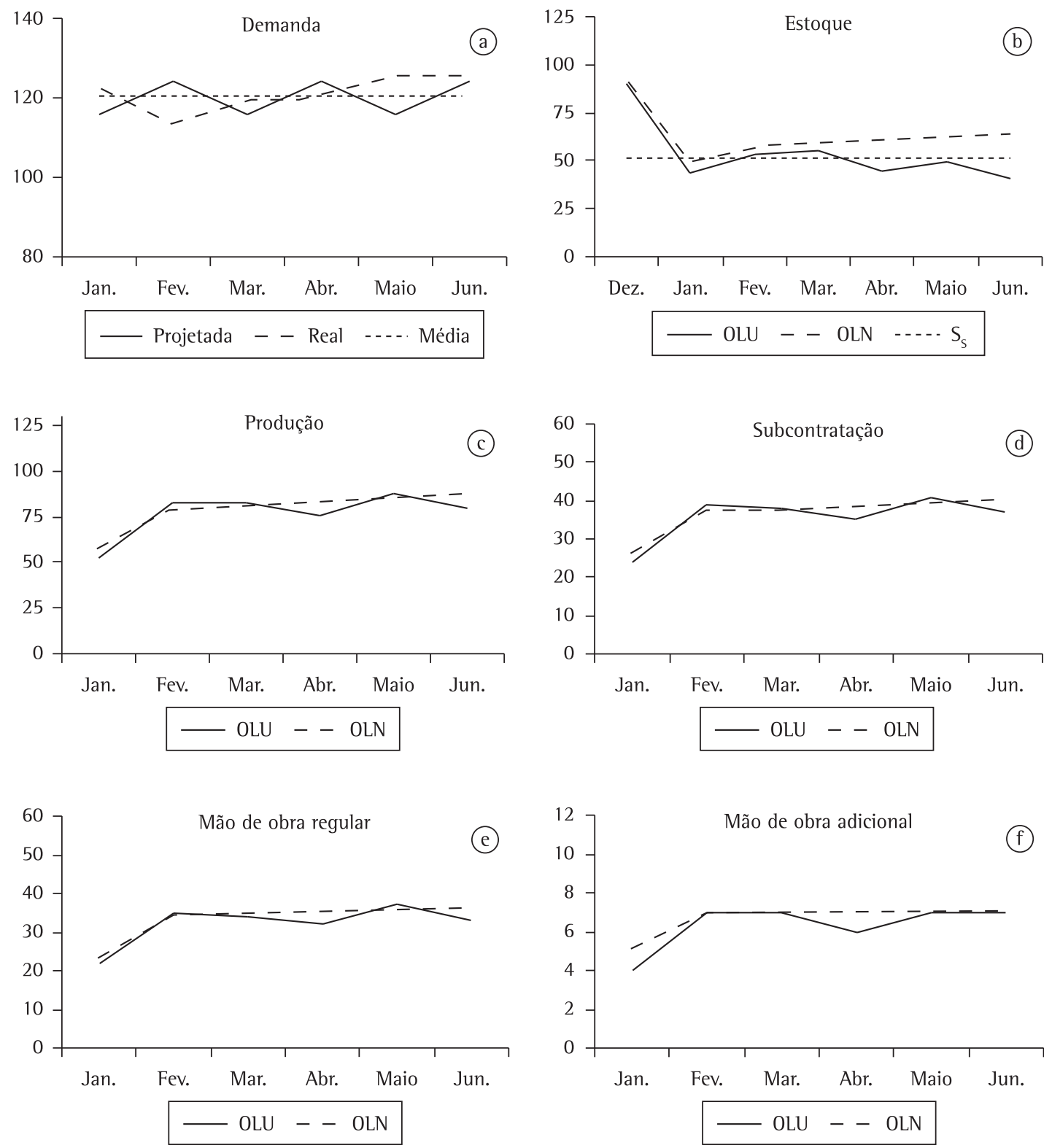

Figura 2. Demanda projetada e trajetórias ótimas - cenário 1.

As Figuras $2 \mathrm{~b}$ a $2 \mathrm{f}$ ilustram os comportamentos das demais variáveis de decisão geradas a partir da aplicação das duas abordagens. Comparando-se estas trajetórias, pode-se observar que: no caso dos níveis de estoques, vide Figura $2 \mathrm{~b}$, a aplicação do procedimento OLN é responsável pela formação de estoques (vide linha tracejada), que crescem ao longo dos períodos do horizonte de planejamento. Já os níveis de estoques resultantes da aplicação da abordagem proposta (vide linha cheia) mantêm-se praticamente estáveis em torno do estoque de segurança $S_{S}$ (linha pontilhada). A explicação para as duas características observadas está no processo de atualização da informação, ou seja, no caso da aplicação do procedimento OLN ao PEM a política gerada não sofre qualquer atualização no tempo, assim, para reduzir o risco de não atender a um súbito aumento de demanda (não previsto), a tendência é gerar estoque que permita garantir os 95\% de satisfação do cliente. Por outro lado, a abordagem proposta que incorpora o procedimento OLU para atualização da política de produção ao longo do tempo, permite uma solução de menor risco e, portanto, não geradora de estoques. É 
ainda importante destacar que o nível de estoques gerado pela abordagem proposta depende, como se pode observar a partir da Figura 1, da demanda real $d_{t}$, juntamente com as entradas $\left\{u_{t}^{*}, v_{t}^{*}\right\}$ resultantes da solução do PEM. Assim, o nível de estoque resultante é o valor real observado pelo sistema de estoque, o que explica o fato dele violar o nível de estoque de segurança em alguns períodos.

Prosseguindo, é interessante observar o comportamento das demais variáveis de decisão geradas pelas duas abordagens e ilustradas nas trajetórias das Figuras 2c a 2f. Este comportamento reflete o comportamento da demanda real, pois se deve ter em mente que, no caso particular do procedimento OLN aplicado ao PEM, a demanda real foi utilizada no lugar da demanda projetada. Uma vez que a intensidade da magnitude das flutuações de demanda é pequena $\left(\sigma_{d}=4\right)$, as variáveis de decisão diretas $\left(u_{t}, v_{t}\right)$ e indiretas $\left(w_{t}\right.$, $z_{t}$ ), resultantes da aplicação das duas abordagens, apresentam um comportamento muito próximo embora tenham objetivos distintos. De fato, enquanto a política OLN é orientada para a geração de estoques, a política OLU é orientada para o atendimento da demanda real, buscando a redução dos níveis de estoques.

Os resultados acima, relacionados às políticas ótimas, são mais bem percebidos quando se compara individualmente cada um dos custos resultantes. Assim procedendo, é possível verificar que a política OLU apresenta um impacto menor nos custos diretos da empresa, o que implica em maior lucratividade. De fato, analisando os valores financeiros, tem-se que o custo total incorrido pela abordagem OLN foi de $\$ 1,565$ milhões, enquanto pelo OLU foi de $\$ 1,245.344$ milhões, representando neste caso uma redução de 20,4\% no custo da política OLN. A Tabela 1 mostra os custos específicos relacionados às variáveis de decisão do problema.

É interessante observar que, refletindo o comportamento das trajetórias das variáveis de decisão, ilustradas na Figura 2, os custos com produção, subcontratação, mão de obra e mão de obra adicional ficaram bem próximos. No entanto, a maior diferença está nos custos com estoques, como mostra a Tabela 1.

\subsubsection{Cenário 2: Flutuação de demanda com} desvio padrão $\sigma_{\mathrm{d}}=20$

Neste cenário, a demanda real $d_{t}$ apresenta forte variabilidade nos níveis de flutuação ao longo do tempo. Note-se que, o desvio padrão neste caso é cinco vezes maior que aquele adotado no cenário anterior. As trajetórias da demanda real $d_{\mathrm{t}} \mathrm{e}$ demanda projetada $\hat{d}_{t}$ estão ilustradas na Figura 3a, nos moldes discutidos no cenário 1 .

As trajetórias ótimas das variáveis de decisão, obtidas pela aplicação da abordagem proposta e do procedimento OLN, estão ilustradas pelas Figuras $3 b$ a 3f. É interessante observar que, embora a natureza das variáveis - subcontratação, mão de obra e hora extra - seja essencialmente determinística, as políticas ótimas associadas com estas variáveis já apresentam diferenças mais significativas do que no caso anterior. De fato, a falta de atualização periódica da abordagem OLN faz com que os níveis destas variáveis oscilem próximo aos seus limites máximos permitidos. Enquanto que a política de estoques apresenta um comportamento semelhante ao do caso anterior, embora crescendo a níveis muito elevados em razão da alta variabilidade da demanda projetada. Com efeito, ao se aplicar à abordagem OLN, os níveis de estoques ao longo dos meses crescem de forma muito expressiva, o que significa uma política totalmente orientada a gerar produtos para estoque. Contrastando a isto, a abordagem OLU consegue manter níveis de estoque bem mais baixos que os obtidos pelo procedimento OLN, como se constata na Figura 3b.

A diferença entre as duas políticas pode ser mais bem avaliada a partir da análise dos ganhos financeiros obtidos. Neste caso, o custo obtido a partir da aplicação da abordagem OLU foi de $\$ 1,224.244$ milhões, enquanto que o custo incorrido na aplicação do procedimento OLN foi de $\$ 3,168.795$ milhões. Isto significa que o custo para operar o sistema produtivo com a política OLU apresenta uma redução em torno de 61,4\% em relação ao custo de aplicação da política OLN. A Tabela 2 mostra como ficam distribuídos os custos específicos das cinco variáveis de decisão do problema.

Tabela 1. Custos de operação dos procedimentos OLU e OLN para o cenário 1.

\begin{tabular}{crcccc}
\hline Custos & Estoque & Produção & Subcontratação & Mão de obra & Mão de obra adicional \\
\hline OLU & 876.080 & 182.450 & 116.620 & 63.352 & 6.842 \\
OLN & $1,171.400$ & 194.480 & 124.300 & 67.526 & 7.294 \\
\hline
\end{tabular}



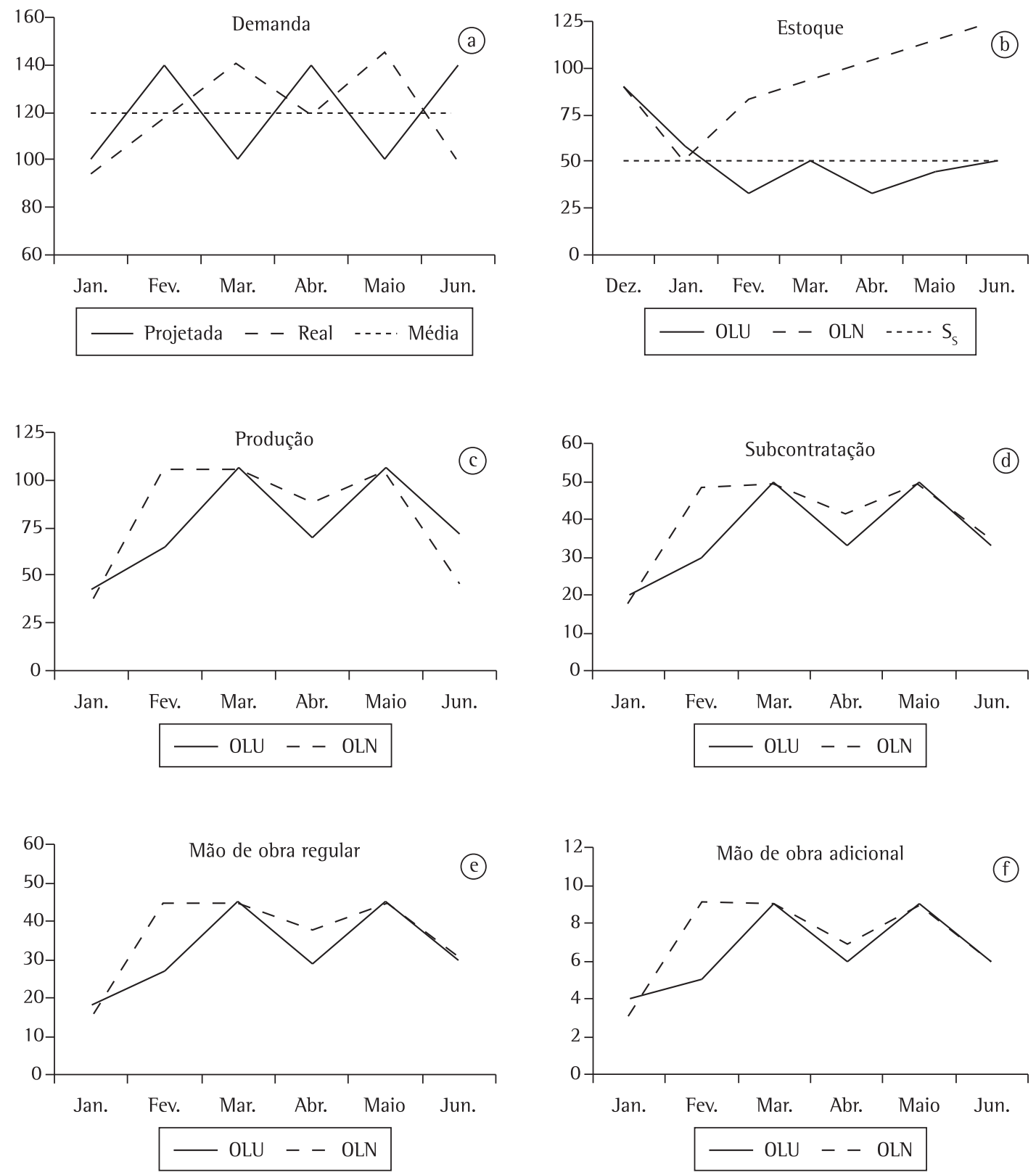

Figure 3. Demanda projetada e trajetórias ótimas - cenário 2.

Tabela 2. Custos de operação dos procedimentos OLU e OLN para o cenário 2.

\begin{tabular}{cccccc}
\hline Custos & Estoque & Produção & Subcontratação & Mão de obra & Mão de obra adicional \\
\hline OLU & 826.680 & 196.440 & 125.550 & 68.208 & 7.366 \\
OLN & $2,683.900$ & 239.590 & 153.130 & 83.190 & 8.985 \\
\hline
\end{tabular}

\subsubsection{Comparando os cenários}

Comparando-se os dois cenários, percebem-se muitas similaridades quanto ao comportamento das trajetórias das variáveis de decisão e seus custos associados. Independentemente da magnitude da variabilidade de demanda, pode-se constatar que a política baseada na abordagem proposta apresentou sempre menores custos e também menor nível de estoque ao longo do tempo do que a política gerada pelo procedimento OLN. Este melhor desempenho é explicado no fato da abordagem proposta 
permitir revisões periódicas durante a geração de sua solução ótima. De fato, enquanto a política OLU é mensalmente atualizada, com informação sobre o nível de estoque corrente (influenciado pela demanda real), a política OLN é resultado de uma solução estática no tempo, em que a única informação sobre o estado da equação de balanço de estoque é aquela obtida no início do mês de janeiro, ou seja, $x_{0}=90$. Como consequência da pouca visibilidade da demanda em períodos futuros, a abordagem OLN tende a gerar níveis de estoque adicionais ao estoque de segurança fixado pela gerência, de modo a compensar esta deficiência. Com isto, a abordagem OLN pretende reduzir o risco de falta do produto em decorrência de aumentos abruptos de demanda, especialmente próximo aos períodos finais do horizonte de planejamento.

\section{Conclusão}

Um problema estocástico multiperíodo de planejamento de produção com restrições nas variáveis de decisão foi analisado. Desde que uma solução ótima global é muito difícil de ser obtida, por razões de dimensionalidade, das restrições explícitas e da natureza aleatória do problema, optou-se por soluções subótimas. A abordagem subótima proposta neste estudo considerou um problema determinístico equivalente ao problema original, em que se procurou preservar os principais momentos estatísticos das variáveis aleatórias envolvidas, sendo este denominado de problema estendido da média. Uma adaptação do procedimento clássico OLU foi empregada para resolver o problema equivalente. A motivação principal foi tornar a abordagem de fácil implementação computacional, além de permitir a atualização periódica na política ótima gerada. Para ilustrar a aplicabilidade da metodologia, foi proposto um exemplo de um problema de planejamento agregado da produção, cujo objetivo era desenvolver um plano ótimo para uma família agregada de produtos num horizonte semestral. Em seguida, foram analisados dois cenários, que levaram em conta suave e intensa variabilidade dos níveis de flutuação da demanda ao longo do tempo. Neste contexto, a política ótima gerada pela abordagem proposta foi comparada com a política malhaaberta gerada pelo procedimento OLN. Em ambos os cenários, a política com atualizações periódicas apresentou melhor desempenho, conseguindo não só equilibrar melhor o conflito entre estocar e entregar produtos nos prazos mas também reduzir os custos operacionais, aumentando assim a lucratividade do negócio.
Como futuros desdobramentos deste trabalho, consideram-se: (1) geração de exemplos aleatórios a partir do modelo, objetivando uma análise aprofundada do grau de sensibilidade deste em relação às suas variáveis de decisão. Assim, a ideia é criar faixas superiores e inferiores relacionadas com a variação da demanda (pois ela é uma variável aleatória independente no modelo) e aplicar a abordagem estudada para desenvolver diferentes cenários relacionados com estas faixas. A região gerada deverá permitir a análise e avaliação do desempenho da abordagem com respeito à sua aplicabilidade ao modelo proposto. (2) aplicação de procedimentos subótimos mais sofisticados como, por exemplo, as técnicas partial open-loop feedback controle $m$-measurement feedback policy (BERTESEKAS, 2000) com o objetivo de gerar soluções adaptativas que incorporarem um número maior de medidas sobre o estado do sistema real; e (3) realização de análise comparativa entre diferentes abordagens, tais como as mencionados acima, no sentido de comprovar seus desempenhos adaptativos. Para este fim, haverá necessidade de redução da dimensionalidade do PEM, de modo que se possa aplicar o algoritmo de programação dinâmica estocástica e assim gerar uma solução ótima global que servirá como referência comparativa para os demais resultados. Um caminho interessante a ser investigado são os modelos aproximativos do algoritmo de programação dinâmica que prometem resolver o problema da maldição da dimensionalidade, vide Powell (2007).

\section{Referências}

BERTESEKAS, D. P. Dynamic programming and optimal control. Belmont: Athena Scientific, 2000. v. 1.

BERTESEKAS, D. P. Dynamic programming and stochastic control, mathematics in science and engineering. New York: Academic Press, 1976. v. 125.

BITRAN, G. R.; YANASSE, H. Deterministic approximation to stochastic production problem. Operations Research, v. 32, n. 5, p. 999-1018, 1984.

CHENG, L.; SUBRAHMANIAN, E.; WESTERBERG, A. W. A comparison of optimal and stochastic programming from a formulation and computation perspective. Computers and Chemical Engineering, v. 29, n. 15, p. 149-164, 2004.

FEIRING, B. R.; SASTRI, T. Improving production planning by utilizing stochastic programming. Computers Industrial Engineering, v. 19, n. 14, p. 53-56, 1990.

GARDNERA, W. A.; NAPOLITANO, A.; PAURAC, L. Cyclostationarity: half a century of research. Signal Processing, v. 86, n. 4, p. 639-697, 2006.

GRAMA, A. et al. Introduction to parallel computing. 2 ed. London: Addison Wesley, 2003. 
GRAVES, S. C. A. Single-item inventory model for a nonstationary demand process. Manufacturing and service operations management, v. 1, n. 1, p. 50-61, 1999.

HACKMAN, S. et al. A stochastic production planning model. Georgia: Technical Report, 2002.

HAX, A. C.; CANDEA, D. Production and inventory management. New Jersey: Prentice-Hall, 1984.

HOLT, C. C. et al. Planning production inventory and workforce. New Jersey: Prentice-Hall, 1960.

KLEINDORFER, P. R. Stochastic control models in management science: theory and computation. Wiley: Applied Optimal Control, 1978. p. 69-88. (TIMS Studies in the Management Science, v. 9).

KLEINDORFER, P. R. et al. Discrete optimal control of production plans. Management Science, v. 22, n. 3, p. 261-273, 1975.

LEVl, R. et al. Approximation algorithms for stochastic inventory control models. Manufacturing and Service Operations Management, v. 7, n. 1, p. 81-99, 2005.

ORTEGA, M.; LIN, L. Control theory applications to the production-inventory problem: a review. International Journal of Production Research, v. 42, n. 11, p. 2303-2322, 2004.

ÖZDAMAR, L.; BOZYEL, M. A.; BIRBIL, S. 1. A. Hierarchical decision support system for production planning. European Journal of Operational Research, v. 104, n. 3, p. 403-422, 1998

PAPOULIS A. Probability, random variables, and stochastic processes. 3 ed. Singapore: McGraw-Hill, 1991.

PEKELMAN, D.; RAUSSER, G. C. Adaptive control: survey of methods and applications. Wiley: Applied Optimal
Control, 1978. p. 89-120. (TIMS Studies in the Management Science, v. 9).

PEREIRA, F. L.; SOUSA, J. B. On the receding horizon hierarchical optimal control of manufacturing systems. Journal of Intelligent Manufacturing, v. 8, n. 5, p. 425-433, 1997.

POWELL, W. B. Approximate dynamic programming: solving the course of dimensionality. New Jersey: John Wiley and Sons, 2007.

RANTALA J.; KOIVISTO, $\mathrm{H}$. Production planning under stochastic time-varying demand: flexible service approach. In: IFAC WORLD CONGRESS, 2005. Proceedings... Praga: Elsevier Science, 2006.

SHEN, R. F. C. Aggregate production planning by stochastic control. European Journal of Operations Research, v. 73, n. 2, p. 346-359, 1994.

SILVA FILHO, O. S.; CEZARINO, W. Geração de planos de produção via otimização seqüencial subótima. Gestão e Produção, v. 14, n. 2, p. 239-252, 2007.

YILDIRIM, I.; BARIS, T.; KARAESMEN, F. A. Multi-period stochastic production planning and sourcing problem with service level constraints. OR Spectrum, v. 27, n. 2-3, p. 471-489, 2005.

\section{Agradecimentos}

Aos referees, pelas recomendações de modificação no texto sugeridas, e ao CNPq, pelo processo $n^{\circ}$. 500202/2003-6.

\title{
Adaptive approach applied to aggregate production planning under uncertainties
}

\begin{abstract}
A problem of aggregate production planning, with uncertainty about the fluctuation in demand, is formulated by a stochastic optimization model, with quadratic criterion and linear constraints. Difficulties in finding a global optimum solution to the problem lead to the proposal of an adaptive approach which is easy to implement computationally and which is based on the formulation of a deterministic equivalent problem, the solution for which is periodically reviewed through a classical procedure of literature. An example, where the inventory balance system is subject to weak and strong variability in actual demand, is employed to analyze the behavior of the proposed approach. Finally, the results provided by the proposed approach are compared with another suboptimal approach, the main characteristic of which is not allowing periodic reviews.
\end{abstract}

Keywords

Inventory control. Planning. Optimization. Stochastic process. Simulation. 